Rosane Do Rocio CoRdeiro Thiel ${ }^{1}$

MIRIAM DAMBROS ${ }^{2}$

Paulo César Rodrigues Palma ${ }^{3}$

MARCELO Thiel ${ }^{4}$

CÁSSIO Luís ZANeTtin RICCETTO ${ }^{5}$

MaRIa de FÁtIMA RAMOS ${ }^{6}$

\section{Tradução para português, adaptação cultural e validação do Female Sexual Function Index}

\author{
Translation into Portuguese, cross-national adaptation and validation \\ of the Female Sexual Function Index
}

Artigos originais

Palavras-chave

Questionários Saúde da mulher Disfunções sexuais psicogênicas Reprodutibilidade dos testes

Estudos de validação Tradução (Produto)

Keywords

Questionnaires

Women's health Sexual dysfunctions, psychological Reproducibility of results Validation studies Translations

\section{Resumo}

OBJETIVO: traduzir para o português, adaptar culturalmente e validar o questionário Female Sexual Function Index (FSFI). MÉTODOS: dois tradutores brasileiros, cientes dos objetivos desta pesquisa, preparam duas versões do FSFI para o português, as quais foram retro-traduzidas por outros dois tradutores ingleses. As diferenças foram harmonizadas e prétestadas em um estudo piloto. As versões finais do FSFI e de outro questionário, o Short-Form Health Survey, já vertido e publicado em português, foram simultaneamente administradas a cem pacientes. Foram testadas as propriedades psicométricas do FSFI, como confiabilidade (consistência interna e teste-reteste) e validades de construto. $\bigcirc$ reteste foi realizado após quatro semanas, a partir da primeira entrevista. RESULTADOS: o processo de adaptação cultural não alterou a versão em português do FSFI comparado ao original. $\bigcirc$ alfa de Cronbach padronizado do questionário foi 0,96; avaliado por domínios, variou de 0,31 a 0,97. Como medida de confiabilidade teste-reteste, foi aplicado o coeficiente de correlação intra-classes, que foi considerado forte e idêntico $(1,0)$. $\bigcirc$ coeficiente de correlação de Pearson entre o FSFI e o Short-Form Health Survey foi positivo, mas fraco na maioria dos domínios afins, variando de 0,017 a 0,036. CONCLUSÕES: a versão do FSFI foi traduzida para o português e adaptada culturalmente e é válida para avaliação da resposta sexual das mulheres brasileiras.

\section{Abstract}

PURPOSE: to translate from English into Portuguese, adapt culturally and validate the Female Sexual Function Index (FSFI). METHODS: knowing the objectives of this research, two Brazilian translators have prepared a version each from the FSFI into Portuguese. Both versions have then been retro-translated into English by two English translators. After harmonizing the differences, they have been pre-tested in a pilot study. The final versions from the FSFI and from another questionnaire, the Short-Form Health Survey, which had already been translated and published in Portuguese, have then been simultaneously administered to one hundred patients, to test the FSFI psychometric proprieties concerning reliability (internal consistency and testing-retesting) and construct validity. Retesting was done after four weeks from the first interview. RESULTS: the process of cultural adaptation has not altered the Portuguese version of the FSFI, as compared to the original. The FSFI standardized Cronbach alpha was 0.96 , and the evaluation by domains has varied from 0.31 to 0.97 . As a measure of test-retest confidentiality, it was applied the intra-class coefficient, which has been considered strong and identical (1.0). Pearson's correlation coefficient between the FSFI and the Short-Form Health Survey was positive, but weak in most of the interrelated domains, varying from 0.017 to 0.036 . CONCLUSIONS: the FSFI English version has been translated into Portuguese and culturally adapted, being reliable to evaluate the sexual response of Brazilian women.
Correspondência:

Rosane do Rocio Cordeiro Thiel Rua Germânia, 270, apto. 23, Edifício Ilha das Rocas - Bonfim CEP 13070-770 - Campinas/SP Fone: (19) 3385-8050 E-mail: rosanethie|@uol.com.br ou thiel7@uol.com.br Home page: www.marcelothiel.med.br

Recebido

14/2/08

Departamento de Cirurgia da Divisão de Urologia da Faculdade de Ciências Médicas da Universidade Estadual de Campinas UNICAMP - Campinas (SP), Brasil.

' Pós-Graduando, Nível Doutorado, do Departamento de Cirurgia da Universidade Estadual de Campinas - UNICAMP - Campinas (SP), Brasil.

${ }^{2}$ Chefe da Divisão de Urogeriatria da Escola Paulista de Medicina da Universidade Federal de São Paulo - UNIFESP - São Paulo (SP), Brasil.

${ }^{3}$ Professor Titular de Urologia da Universidade Estadual de Campinas - UNICAMP - Campinas (SP), Brasil.

4 Pesquisador, Nível Pós-Doutorado, da Divisão de Urogeriatria da Escola Paulista de Medicina da Universidade Federal de São Paulo - UNIFESP - São Paulo (SP), Brasil.

${ }^{5}$ Livre-docente, Professor do Programa de Pós-graduação em Cirurgia da Universidade Estadual de Campinas - UNICAMP - Campinas (SP), Brasil. 


\section{Introdução}

A função sexual adequada é um fator importante de satisfação e qualidade de vida geral; mesmo assim, a disfunção sexual feminina continua altamente prevalente ${ }^{1}$. Dados do National Health and Social Life Survey (NHSLS) referem que entre 30 e $50 \%$ de mulheres americanas têm algum tipo de disfunção sexual ${ }^{2}$. Entre as brasileiras, $30 \%$ apresentam alguma dificuldade sexual, e apenas 5\% procuram tratamento ${ }^{3}$. A Organização Mundial de Saúde (OMS) reconhece a disfunção sexual como problema de Saúde Pública e recomenda sua investigação por causar importantes alterações na qualidade de vida ${ }^{4,5}$.

A função sexual feminina resulta da interação de vários fatores, e os aspectos subjetivos tornam sua investigação mais complexa ${ }^{6}$. O aumento do interesse pelo estudo da sexualidade tem favorecido avanços nessa área, mas ainda há escassez de dados confiáveis e muitas pesquisas são realizadas de forma inadequada ${ }^{7}$. Há grande diversidade de métodos, bem como de tamanho de amostras. Além disso, algumas informações não podem ser generalizadas para toda população feminina, porque as diferenças culturais e sociais afetam significativamente o comportamento e a resposta sexual ${ }^{7,8}$. O uso de questionários validados pode ser um facilitador internacional para o avanço das pesquisas, por obedecerem a regras rígidas de tradução e confiabilidade e por favorecerem trabalhos multicêntricos que possibilitem a comparação dos resultados ${ }^{8}$.

Entre os questionários genéricos de avaliação de saúde, o mais utilizado é o The Medical Outcomes Study 36-item Short Form Health Survey (MOS SF-36 Health Survey $)^{9}$. Instrumentos genéricos de qualidade de vida desenvolvidos para investigar a saúde e o bem-estar geral são insuficientes para avaliar a função sexual e não fornecem informações necessárias para estabelecer propostas terapêuticas. Instrumentos de avaliação da função sexual poderiam acessar aspectos de desordens específicas, além da opinião do indivíduo sobre o impacto do problema em sua qualidade de vida ${ }^{10}$.

Atualmente, discute-se a necessidade de empregar instrumentos específicos e objetivos para avaliar a função sexual feminina, por serem mais sensíveis a aspectos vinculares, emocionais e psicossociais, como o Female Sexual Function Index (FSFI $)^{11,12}$. Pesquisas brasileiras também carecem de instrumento de avaliação específico e validado em português ${ }^{13,14}$. Consulta aos bancos de dados Medline e Lilacs, de janeiro de 2003 a fevereiro de 2008, localizou três questionários que validaram o FSFI para o português. No entanto, um deles foi validado em mulheres grávidas ${ }^{15}$, outro em mulheres inférteis ou de casal infértil ${ }^{16}$ e o último com amostra de dez pacientes ${ }^{17}$. Gravidez ou infertilidade representam vieses na avaliação da sexualidade, devido às alterações que ocorrem nestas condições ${ }^{18,19}$.
O FSFI, ou índice da função sexual feminina, é um questionário construído e validado na língua inglesa, específico e multidimensional, para avaliar a resposta sexual feminina, acessando seus domínios ${ }^{20}$. $\mathrm{O}$ instrumento foi localizado após consultas aos bancos de dados, por ser muito utilizado em pesquisas clínicas e ter propriedades psicométricas válidas. O questionário é composto por 19 questões, que informam sobre cinco domínios da resposta sexual: desejo e estímulo subjetivo, lubrificação, orgasmo, satisfação e dor ou desconforto. Pontuações individuais são obtidas pela soma dos itens que compreendem cada domínio (escore simples), que são multiplicadas pelo fator desse domínio e fornecem o escore ponderado. A pontuação final (escore total: mínimo de 2 e máximo de 36) é obtida pela soma dos escores ponderados de cada domínio ${ }^{20}$.

O MOS SF-36 Health Survey é um questionário multidimensional, formado por 36 itens reunidos em dois componentes: físico e mental. Cada um destes componentes é formado por quatro domínios, que, por sua vez, se constituem de itens que avaliam uma mesma área da vida dos pacientes. O componente físico é composto pelos seguintes domínios: "capacidade funcional”, "aspectos físicos", "dor", "estado geral de saúde”. Já o componente mental abrange domínios como "vitalidade", "aspectos sociais", "aspectos emocionais" e "saúde mental". Existe, ainda, uma questão de avaliação comparativa entre as condições atuais de saúde e as de há um ano ${ }^{8}$. Pode-se obter um escore final de cada domínio que varia de 0 a 100 , e, como no FSFI, quanto maior a pontuação, melhor é o estado de saúde avaliado. Entretanto, o MOS SF-36 Health Survey não tem avaliação específica da resposta sexual feminina. O objetivo deste estudo é traduzir para o português, adaptar culturalmente e validar o FSFI.

\section{Métodos}

\section{Pacientes}

Realizou-se estudo observacional (tipo coorte transversal) em cem mulheres atendidas no Serviço de Urologia da Universidade São Francisco (USF), de Bragança Paulista, em São Paulo, no período de junho a dezembro de 2006. O tamanho da amostra foi determinado admitindo-se uma correlação mínima de -0,30 entre os domínios dos dois instrumentos (FSFI e MOS SF-36 Health Survey) e fixando-se um erro tipo I de $5 \%$ e um erro tipo II de $10 \%$. Foram excluídas pacientes sem parceiro, em período gestacional ou de amamentação. Excluíram-se mulheres com idade inferior a 18 anos, que usavam antidepressivos ou com doenças incapacitantes que impediam o ato sexual. Não se delimitou a idade máxima ou se exigiu relacionamento estável. Todas as pacientes concordaram em participar do estudo e assinaram o termo de consentimento 
livre e esclarecido. O estudo foi aprovado pelo comitê de ética em pesquisa da Faculdade de Ciências Médicas da Universidade Estadual de Campinas (Unicamp).

\section{Variáveis clínicas e instrumentos}

Após consulta prévia no Serviço de Urologia, foram coletadas informações sociodemográficas (idade, estado civil, salário e grau de escolaridade), representadas na Tabela 1, e clínicas (hipertensão, diabetes, menopausa, atividade física de rotina e frequiência da atividade sexual), que aparecem na Tabela 2. Na mesma entrevista, foram administradas a versão final do FSFI e a versão do MOS SF-36 Health Survey validada para o português ${ }^{3}$, com o objetivo de avaliar as correlações entre os domínios afins dos dois questionários.

\section{Tradução e adaptação cultural}

O processo de tradução e adaptação cultural seguiu $\mathrm{s}$ normas publicadas e orientações técnicas referidas para adaptação de questionários ${ }^{21}$. A tradução do FSFI original foi realizada por dois professores de inglês, brasileiros, cientes dos objetivos da pesquisa e fluentes no idioma inglês. As duas traduções foram comparadas e obteve-se uma tradução consensual (T1). Esta última foi vertida para o inglês por dois cidadãos ingleses que vivem no Brasil, fluentes nas duas línguas. As duas versões originadas desta etapa foram também comparadas e harmonizadas em uma versão

Tabela 1 - Dados sociodemográficos (idade, estado civil, renda e grau de escolaridade) expressos em número absoluto e porcentagem

\begin{tabular}{|c|c|c|}
\hline Tipo de informaç̃̃o & Número absoluto & $\%$ \\
\hline \multicolumn{3}{|l|}{ Idade } \\
\hline Média & 27,25 & \\
\hline Ampliłude (mínima-máxima) & $19-69$ & \\
\hline Desvio padrão & 8,97 & \\
\hline \multicolumn{3}{|l|}{ Estado civil } \\
\hline Solteira & 75 & 75 \\
\hline Casada & 21 & 21 \\
\hline Divorciada & 2 & 2 \\
\hline Separada & 1 & 1 \\
\hline Desquitada & 1 & 1 \\
\hline \multicolumn{3}{|l|}{ Salários mínimos } \\
\hline Média & 1,83 & \\
\hline Amplitude (mínima-máxima) & $0-11$ & \\
\hline Desvio padrão & 2,79 & \\
\hline \multicolumn{3}{|l|}{ Grau de escolaridade } \\
\hline Fundamental & 11 & 11 \\
\hline Médio & 31 & 31 \\
\hline Superior incompleto & 33 & 33 \\
\hline Superior completo & 25 & 25 \\
\hline
\end{tabular}

A idade e a renda (número de salários mínimos) são informadas nas formas de média, variação (amplitude) e desvio padrão. Os diferentes graus de escolaridade e o estado civil das mulheres entrevistadas são informados em número e porcentagem. final de retro-tradução (V1), que se mostrou gramatical e semanticamente equivalente ao instrumento original. Assim, até esta fase, a T1 foi aceita como a tradução do FSFI para o português. As equivalências idiomáticas e culturais de T1 foram analisadas por um comitê de dez juízes bilíngües, todos profissionais da área da Saúde. Após revisão das considerações desses juízes, obteve-se a tradução T2, que foi pré-testada em estudo piloto com dez pacientes. Como as questões da T2 apresentaram índice de compreensão apropriado, a tradução foi aplicada à amostra populacional. O FSFI foi auto-administrado.

\section{Validação e confiabilidade}

A consistência interna do FSFI foi calculada com base nos escores finais obtidos dos questionários preenchidos na primeira entrevista das cem pacientes. A avaliação

Tabela 2 - Dados clínicos

\begin{tabular}{|c|c|c|}
\hline Tipo de informação & Número absoluto & $\%$ \\
\hline \multicolumn{3}{|l|}{ Número de gestações } \\
\hline Média & 0,5 & \\
\hline Ampliłude (mínima-máxima) & $0-5$ & \\
\hline Desvio padrão & 1,2 & \\
\hline \multicolumn{3}{|l|}{ Cirurgias prévias } \\
\hline Abdominal e pélvica & 1 & \\
\hline Abdominal & 3 & \\
\hline Pelve & 7 & \\
\hline Períneo & 3 & \\
\hline Nenhuma & 86 & \\
\hline \multicolumn{3}{|l|}{ Hipertensão } \\
\hline Sim & 6 & 6 \\
\hline Não & 94 & 94 \\
\hline \multicolumn{3}{|l|}{ Diabetes } \\
\hline Sim & 2 & 2 \\
\hline Não & 98 & 98 \\
\hline \multicolumn{3}{|l|}{ Outras doenças } \\
\hline Enxaqueca & 1 & 1 \\
\hline Hipotireodismo ou dislipidemia & 1 & 1 \\
\hline \multicolumn{3}{|l|}{ Menopausa } \\
\hline $\operatorname{Sim}$ & 5 & 5 \\
\hline Não & 95 & 95 \\
\hline \multicolumn{3}{|l|}{ Atividade física de rotina } \\
\hline Sim & 29 & 29 \\
\hline Não & 26 & 26 \\
\hline Não respondeu & 45 & 45 \\
\hline \multicolumn{3}{|l|}{$\begin{array}{l}\text { Frequiência de atividade sexual no } \\
\text { período de } 4 \text { semanas }\end{array}$} \\
\hline 1 & 14 & 14 \\
\hline 2 & 16 & 16 \\
\hline 3 & 24 & 24 \\
\hline 4 & 46 & 46 \\
\hline
\end{tabular}

Informam-se o número e a porcentagem de mulheres com hipertensão, diabetes e outras comorbidades. O mesmo ocorre com menopausa, atividade física de rotina e freqüência de atividade sexual no período de quatro semanas. 
da reprodutibilidade do FSFI foi realizada após quatro semanas, nas mesmas cem pacientes. A validade de construto foi avaliada pela correlação entre os resultados obtidos nos domínios do FSFI e do MOS SF-36 Health Survey, aplicados na primeira entrevista a cem pacientes. A validade concorrente foi avaliada pela associação dos domínios do FSFI com algumas variáveis clínicas selecionadas: idade, estado civil, grau de escolaridade e atividade física de rotina.

\section{Análise dos dados}

A análise estatística descritiva utilizou freqüência, média ( \pm desvio padrão, dp) e mediana (intervalo). A medida de confiabilidade ou consistência interna foi medida com o coeficiente de Cronbach padronizado (valor mínimo de 0,70 ), que avalia a homogeneidade de uma pergunta com as demais do questionário. O teste-reteste (mínimo de 0,90$)$ calcula o coeficiente de correlação intraclasses e permite dizer se a ferramenta estudada é confiável ou não. A validade de construto foi analisada por meio do coeficiente de correlação linear de Pearson, e a validade concorrente foi analisada pelos testes Mann-Whitney e Kruskal-Wallis. Na comparação das variáveis contínuas ou ordenáveis entre dois grupos independentes, foi utilizado o teste Mann-Whitney e, para três ou mais grupos, o teste de Kruskal-Wallis. O nível de significância adotado foi de $5 \%$. O questionário pode ser obtido através de contato com a autora (rosanethiel@uol.com.br) ou no endereço: www. marcelothiel.med.br, no link Questionário de Avaliação da Resposta Sexual Feminina.

\section{Resultados}

Foram entrevistadas cem mulheres com média de idade de 27,3 anos (dp=9), mediana de 21 anos (- 69 anos). A maioria delas $(75 \%)$ era solteira, mas todas com parceria estável. Quanto à renda, a média foi de 1,8 (0-11) salários mínimos. Todas as pacientes tinham alguma atividade, como trabalho ou estudo (Tabela 1). Os dados clínicos são apresentados na Tabela 2. Com relação ao grau de escolaridade, $11 \%$ tinham ensino fundamental, $31 \%$ ensino médio, $33 \%$ superior incompleto e $25 \%$ superior completo (Tabela 3).

Achados da combinação dos dois questionários com varáveis clínicas verificaram correlação moderada $(-0,48, \mathrm{p}<0,001)$ da idade com o domínio "capacidade funcional” (MOS SF-36 Health Survey), o que significa que com o aumento da idade, diminui, numa proporção moderada, o escore da capacidade funcional. O mesmo foi observado entre a idade e o domínio "dor" (MOS SF-36 Health Survey), que apresentou correlação negativa, de fraca intensidade $(-0,29, \mathrm{p}=0,003)$, demonstrando que quanto maior a idade, menor a capacidade funcional e menor a referência de dor. Já na análise dos itens do FSFI, a idade tem correlação negativa moderada $(-0,36$, $\mathrm{p}<0,001)$ com o domínio "satisfação", e fraca com o domínio "desejo" $(0,020, p=0,046)$ e com o escore geral do FSFI $(-0,20, p=0,044)$. Também houve correlação positiva fraca $(0,021, \mathrm{p}=0,038)$ entre idade e aspectos físicos (Tabela 4).

O teste de Kruskal-Wallis indicou que, para a variável escolaridade, existem diferenças significativas entre os níveis de escolaridade para alguns domínios (considerar resultados equivalentes para pares não mencionados), conforme mostra a Tabela 3 .

Verificou-se, por exemplo, que pacientes com ensino médio apresentaram escore de dor menor do que aquelas com superior incompleto. No MOS SF-36 Health Survey, também foram encontradas diferenças semelhantes nos domínios "estado geral saúde", "aspectos sociais" e "aspectos emocionais". Com relação ao FSFI, pacientes com instrução fundamental apresentaram escore de excitação (aspecto subjetivo) menor do que aquelas com ensino médio e superior incompleto. O nível de escolaridade também influenciou os domínios "lubrificação", "orgasmo", "satisfação" e "escore geral".

O teste de Mann-Whitney indicou uma diferença significativa entre mulheres que praticavam atividade física ou não, nos domínios "vitalidade" $(\mathrm{p}=0,032)$, "aspectos sociais" ( $p=0,012)$ e "saúde mental" $(p=0,03)$ no MOS SF-36 Health Survey, além do domínio "satisfação" $(\mathrm{p}=0,014)$ do FSFI. Houve diferenças significativas entre os resultados apresentados por mulheres solteiras e casadas no domínio "capacidade funcional" do MOS SF-36 Health

Tabela 3 - Teste de Kruskal-Wallis para correlação entre os dois instrumentos e a variável grau de escolaridade

\begin{tabular}{|c|c|c|}
\hline Domínio & $\mathrm{p}$ & Grupos de acordo com a educação \\
\hline \multicolumn{3}{|c|}{ 1. MOS SF-36 Health Survey } \\
\hline Dor & 0,017 & ensino médio - superior incompleto \\
\hline Estado geral saúde & $<0,001$ & $\begin{array}{l}\text { ensino médio - superior incompleto } \\
\text { ensino médio - superior completo }\end{array}$ \\
\hline Aspectos sociais & $<0,001$ & $\begin{array}{l}\text { ensino médio - superior incompleto } \\
\text { ensino médio - superior completo }\end{array}$ \\
\hline Aspectos emocionais & 0,014 & superior incompleto - superior completo \\
\hline \multicolumn{3}{|l|}{ 2. FSFI } \\
\hline Excitação & 0,038 & $\begin{array}{l}\text { fundamental - médio } \\
\text { fundamental - superior incompleto }\end{array}$ \\
\hline Lubrificação & 0,036 & fundamental - médio \\
\hline Orgasmo & 0,009 & $\begin{array}{l}\text { fundamental - médio } \\
\text { fundamental - superior completo }\end{array}$ \\
\hline Satisfação & 0,019 & $\begin{array}{l}\text { fundamental - médio } \\
\text { fundamental - superior incompleto } \\
\text { fundamental - superior completo }\end{array}$ \\
\hline Escore geral & 0,038 & fundamental - médio \\
\hline
\end{tabular}

p: nível de significância; teste: Kruskal-Wallis; MOS SF-36 Health Survey: The Medical Outcomes Study 36-item Short-Form Health Survey'; FSFI: Female Sexual Function Index ${ }^{20}$. 
Tabela 4 - Coeficientes de correlação entre os dois instrumentos

\begin{tabular}{|c|c|c|c|c|c|c|}
\hline FSFI/MOS SF-36 Health Survey & Capacidade funcional & Dor & Saúde geral & Aspectos sociais & Aspectos emocionais & Saúde mental \\
\hline Lubrificação & $\begin{array}{c}0,26 \\
p=0,009\end{array}$ & $\cdot$ & $\cdot$ & $\cdot$ & - & $\begin{array}{c}0,20 \\
p=0,041\end{array}$ \\
\hline Orgasmo & $\begin{array}{c}0,25 \\
p=0,011\end{array}$ & $\cdot$ & - & $\begin{array}{c}0,21 \\
p=0,033\end{array}$ & $\cdot$ & $\begin{array}{c}0,27 \\
p=0,006\end{array}$ \\
\hline Sutisfação & $\begin{array}{c}0,33 \\
p<0,001\end{array}$ & $\begin{array}{c}0,22 \\
p=0,025\end{array}$ & $\begin{array}{c}0,22 \\
p=0,026\end{array}$ & $\begin{array}{c}0,29 \\
p=0,003\end{array}$ & $\begin{array}{c}0,31 \\
p=0,002\end{array}$ & $\begin{array}{c}0,27 \\
p=0,006\end{array}$ \\
\hline Escore geral & $\begin{array}{c}0,20 \\
p=0,036\end{array}$ & - & - & - & - & $\begin{array}{c}0,23 \\
p=0,017\end{array}$ \\
\hline
\end{tabular}

p: nível de significância; teste: coeficiente de correlação de Pearson; MOS SF-36 HEALTH SURVEY: The Medical Outcomes Study 36 -item Short-Form Health Survey9; FSFI: Female Sexual Function Index ${ }^{20}$; a validade de construto foi avaliada com o coeficiente de correlação de Pearson, que verificou a associação linear do MOS SF-36 HEALTH SURVEY com FSFI. A proporção da semelhança é dada pelo valor absoluto do coeficiente. Quanto mais próximo de 1, maior é a semelhança.

Survey $(p=0,042)$ e entre mulheres solteiras e divorciadas no domínio "aspectos sociais" $(\mathrm{p}=0,032)$.

O coeficiente alfa de Cronbach geral do FSFI, que analisou a consistência interna, foi alto $(0,96)$, o que demonstra a homogeneidade do questionário (Tabela 5).

Considerando os domínios individualmente, o escore variou de 0,31 (satisfação) a 0,97 (lubrificação). O domínio "satisfação", composto pelas questões 14, 15 e 16 , apresentou a menor consistência interna. Isso porque a questão 14 mostrou correlação próxima de zero $(0,03)$ com as questões 15 e 16, mas forte correlação positiva com as demais questões do instrumento (alfa de Cronbach de 0,77$)$. Assim, as respostas das pacientes para as questões 15 e 16 não combinam com as demais respostas do questionário, ou seja, os valores numéricos das respostas destoam das demais questões.

\section{Confiabilidade}

A confiabilidade, teste-reteste, foi avaliada pelo coeficiente de correlação intraclasses. Teste e reteste dos dois instrumentos resultaram idênticos $(1,00)$.

\section{Validade de construto}

O coeficiente de correlação de Pearson, que verificou a validade de construto pela correlação entre domínios do MOS SF-36 Health Survey e FSFI, apresenta os domínios que se correlacionam $(\mathrm{p}<0,05)$ e seus respectivos coeficientes (Tabela 4).

Tabela 5 - Coeficientes de Cronbach, Female Sexual Function Index

\begin{tabular}{lcc}
\hline Domínio & Questões & Coeficiente alfa de Cronbach \\
\hline Desejo & 1,2 & 0,88 \\
Exciłação & $3,4,5$ e 6 & 0,94 \\
Lubrificação & $7,8,9$ e 10 & 0,97 \\
Orgasmo & 11,12 e 13 & 0,94 \\
Satisfação & 14,15 e 16 & 0,31 \\
Dor & 17,18 e 19 & 0,96 \\
Geral & Todas & 0,96 \\
\hline
\end{tabular}

teste: coeficientes alfa de Cronbach; o coeficiente alfa de Cronbach analisou a consistência interna do FSFI. O número de questões que analisa cada domínio é diferente e, para existir consistência interna, o coeficiente deve ser maior que 0,80 .
Os domínios "lubrificação", "orgasmo", "satisfação" e o escore geral do FSFI obtiveram correlação fracamente positiva com a capacidade funcional do MOS SF-36 Health Survey, no entanto, estatisticamente significante $(\mathrm{p}=0,009)$. Sendo assim, quanto maior a capacidade funcional, maiores os escores dos domínios do FSFI.

O domínio "satisfação" (FSFI) apresentou correlação com a maior quantidade de itens relacionados do MOS SF-36 Health Survey, apesar de essa correlação ser fracamente positiva, enquanto saúde mental foi o domínio do MOS SF-36 Health Survey que teve relação com o maior número de domínios do FSFI, embora ela tenha sido fracamente positiva.

Nenhum domínio dos dois questionários relacionou-se de forma fortemente positiva, o que significa que nenhum escore dos domínios de um dos instrumentos altera significativamente algum escore do outro.

\section{Discussão}

A teoria psicométrica sugere para avaliação das disfunções sexuais femininas, em pesquisas clínicas, instrumentos auto-administráveis que fornecem resultados mais específicos e confiáveis ${ }^{10}$. Enquanto diários servem para enumerar eventos e/ou contar freqüências, os questionários possibilitam avaliação mais sofisticada da resposta, porque acessam aspectos subjetivos e favorecem a visão multidimensional $^{10}$.

O FSFI é o primeiro instrumento para avaliação da função sexual feminina validado em português e que cumpre todos os requisitos propostos para o protocolo de validação internacional ${ }^{13,14}$. O instrumento foi aplicado juntamente com outro questionário de qualidade de vida geral (MOS SF-36 Health Survey).

As versões do FSFI validadas em português foram feitas em grupos específicos de mulheres. Uma delas foi empregada em mulheres grávidas ${ }^{15}$. Na literatura, são descritas as alterações psico-fisiológicas neste período que interferem na resposta sexual ${ }^{18}$. A validação do questionário deve abranger a população feminina brasileira, de forma irrestrita e sem sofrer o viés de condições que 
interfiram no ciclo sexual. O mesmo ocorreu na validação feita com mulheres inférteis ou casal infértil ${ }^{16}$. Nestes casos, também há alteração da satisfação pela exigência da concepção, principalmente quando ocorre o ciclo ovulatório $^{19}$. Na última validação, não houve definição das características da amostra, como a idade, e ocorreu com dez pacientes apenas ${ }^{17}$.

Neste estudo, a nova versão foi submetida a cem pacientes que procuraram o Serviço de Uroginecologia com diferentes queixas, exceto de distúrbios sexuais, o que torna o questionário mais representativo que os demais.

O FSFI mostrou-se instrumento de fácil compreensão e aplicação, que variou de dez a 20 minutos. A proposta do instrumento é clara, seus componentes são específicos e permitem definir a população estudada ${ }^{22}$.

A consistência interna do FSFI, medida pelo coeficiente alfa de Cronbach padronizado, foi satisfatória, com índice geral de 0,95 e maior que 0,80 para cada domínio. Foram encontrados valores de 0,88 (domínio “desejo") a 0,97 (domínio "lubrificação"), que confirmam as discussões existentes sobre a divergência entre aspectos subjetivos e objetivos da resposta sexual feminina ${ }^{23}$. Não é uma relação proporcional, como acontece no homem, em que a ereção só existe com desejo. Conforme a nova proposta circular da resposta sexual feminina, uma mulher pode iniciar seu ciclo com excitação e lubrificação, que são respostas objetivas, e somente depois ter desejo, que é subjetivo ${ }^{24}$.

A confiabilidade, medida pelo teste-reteste, mostrou que o FSFI validado é confiável porque o coeficiente foi 1 .

A validade de construto foi avaliada com a aplicação do coeficiente de correlação de Pearson, que verificou a associação linear do MOS SF-36 Health Survey com FSFI. Observou-se em todos os domínios correlação fracamente positiva. Os domínios "saúde mental" e "capacidade funcional" (MOS SF-36 Health Survey) foram os que mais se relacionaram com os domínios do FSFI. A proporção da semelhança do comportamento é dada pelo valor absoluto do coeficiente. Os resultados demonstraram que o MOS SF-36 Health Survey não avalia a resposta sexual, sendo necessário um instrumento específico para esse propósito.

O domínio "satisfação", avaliado por três questões (14, 15 e 16), apresentou a menor consistência $(0,31)$. A questão 14 mostra correlação próxima de zero com os demais itens desse domínio (questões 15 e 16), mas tem forte correlação com as demais questões do instrumento (alfa de Cronbach $=0,77$ ). Aparentemente, as questões 15 (alfa de Cronbach =0,48) e 16 (alfa de Cronbach=0,50) não têm correlação com as demais questões do instrumento.

A questão 14 avalia o grau de satisfação com a quantidade de envolvimento emocional entre a mulher e seu parceiro. Assim, se há envolvimento satisfatório o escore é alto e do contrário ele é baixo. Essa questão se relaciona com as demais do questionário, e os escores confirmam estudos anteriores que ressaltam a importância dos aspectos emocional e relacional para a resposta sexual feminina ${ }^{24}$. Proposta recente do ciclo da resposta sexual é que as mulheres também dependem de fatores externos que podem potencialmente despertar ou disparar o desejo durante a experiência ${ }^{24}$.

Estudo americano com 987 mulheres concluiu que os maiores preditores de problemas sexuais, diminuição do desejo e satisfação feminina são o bem-estar emocional geral e o sentimento de proximidade com o parceiro durante a relação ${ }^{1}$.

Já com relação às questões 15 , que avalia o grau de satisfação na relação sexual com o parceiro, e 16, que avalia o grau de satisfação com a vida sexual em geral, as entrevistadas consideraram aspectos objetivos (lubrificação, dor) da sua resposta sexual, ao contrário do que aconteceu no restante do questionário.

A importância da experiência subjetiva para a excitação feminina tem sido referida em estudos psico-fisiológicos nos últimos 25 anos. Atualmente, comenta-se sobre a freqüente falta de correlação entre excitação sexual subjetiva e objetiva (congestão genital e lubrificação). Essa falta de correlação é referida nas definições revisadas dos subtipos de desordens de excitação feminina ${ }^{25}$.

A falta de dados sobre a condição do parceiro é uma limitação da avaliação deste questionário e não deve passar despercebida aos olhos do investigador ${ }^{26}$.

A amostra avaliada foi de mulheres jovens e, na maioria, com grau de instrução elevado. No entanto, não foram avaliados aspectos familiares e religiosos dessas mulheres, que são fatores determinantes de escolhas e comportamentos sexuais. Assim, quando respondem sobre satisfação com sua vida sexual, as pacientes dessa amostra podem ter referido sua dificuldade em ter satisfação por não possuírem vida comum ( $75 \%$ solteiras) ou por não estarem com o parceiro o suficiente para terem intimidade necessária e obterem relações satisfatórias.

Outros dados desse estudo também vêm ao encontro de achados da literatura. Mulheres que praticam atividade física apresentaram melhores escores nos domínios "vitalidade", "aspectos sociais" e "saúde mental" do MOS SF-36 Health Survey e no domínio "satisfação" do FSFI, do que aquelas que são sedentárias. Mulheres com maior nível de escolaridade apresentaram escore de dor mais alto.

\section{Agradecimentos}

A Luciana Maria Guerra e Fabiano Marcelo Fabris, alunos da Faculdade de Medicina da USF, de Bragança Paulista, pelo auxílio na coleta e organização dos dados. 


\section{Referências}

1. Bancroft J, Loftus J, Long JS. Distress about sex: a national survey of women in heterosexual relationships. Arch Sex Behavior. 2003;32(3): 192-208.

2. Laumann EO, Paik A, Rosen RC. Sexual dysfunction in the United Sates: prevalence and predictors. JAMA. 1999;281(6):537-44. Erratum in: JAMA 1999 Apr 7;281(13):1174.

3. Abdo CHN, Oliveira Junior WN, Moreira Junior ED, Fittipaldi JAS. Perfil sexual da população brasileira: resultado do Estudo do Comportamento Sexual (ECOS) do brasileiro. RBM Rev Bras Med. 2002;59(4):250-7.

4. World Health Organization. Gender and reproductive rights [document on the Internet]. Geneva: World Health Organization; 2002 [cited 2007 Aug]. Available from: www.who.int/reproductivehealth/gender/glossary.html

5. Department of Health and Human Services. General's call to action to promote sexual health and responsible sexual behavior [document on the Internet]. Washington, DC; 2001 [cited 2007 Aug]. Available from: http://www.surgeongeneral.gov/library/ sexualhealth/call.pdf

6. Bernhard LA. Sexuality and sexual health care for women. Clin Obstet Gynecol. 2002;45(4):1089-98. Review.

7. Bean JL. Expressions of female sexuality. J Sex Marital Ther. 2002;28 Suppl 1:29-38.

8. Pasqualotto EB, Pasqualotto FF, Sobreiro BP, Lucon AM. Female sexual dysfunction: the important points to remember. Clinics. 2005;60(1):51-60.

9. Ciconelli RM. Tradução para o português e validação do Questionário Genérico de Avaliação de Qualidade de Vida "Medical Outcomes Study 36 - Item Short-Form Health Survey (SF-36)" [tese]. São Paulo: Universidade de São Paulo; 1997.

10. Symonds T, Boolell M, Quirk F. Development of a questionnaire on sexual quality of life in women. J Sex Marital Ther. 2005;31 (5):38597.

11. Althof SE, Rosen RC, DeRogatis L, Corty E, Quirk F, Symonds T. Outcome measurement in female sexual dysfunction clinical trials: review and recommendations. J Sex Marital Ther. 2005;31 (2):153-66.

12. Bertolino V, Bechara AJ. Female sexual dysfunction: a tale of two sides? J Sex Med. 2006;3(2):375.

13. Guillemin F. Measuring health status across cultures. Rheumatol Eur. 1995;24 Suppl 2:102-3.
14. Guillemin F. Cross-cultural adaptation and validation of health status measures. Scand J Rheumatol. 1995;24(2):61-3.

15. Leite APL, Moura EA, Campos AAS, Mattar R, Souza E, Camano L. Validação do índice da função sexual feminina em grávidas brasileiras. Rev Bras Ginecol Obstet. 2007;29(8):396-401.

16. Hentschel H, Alberton DL, Capp E, Goldim JR, Passos EP. Validação do Female Sexual Function Index (FSFI) para uso em português. Rev HCPA. 2007;27(1): 10-4.

17. Pacagnella RC, Vieira EM, Rodrigues Júnior OM, Souza C. Adaptação transcultural do Female Sexual Function Index. Cad Saúde Pública. 2008;24(2):416-26.

18. 18. Malarewicz A, Szymkiewicz J, Rogala J. Sexuality of pregnant women. Ginekol Pol. 2006;77(9):733-9.

19. Shindel AW, Nelson CJ, Naughton CK, Ohebshalom M, Mulhall JP. Sexual function and quality of life in the male partner of infertile couples: prevalence and correlates of dysfunction. J Urol. 2008; 179(3): 1056-9.

20. Rosen R, Brown C, Heiman, J, Leiblum S, Meston C, Shabsigh R, et al. The Female Sexual Function Index (FSFI): a multidimensional self-report instrument for the assessment of female sexual function. J Sex Marital Ther. 2000;26(2):191-208.

21. Guillemin F, Bombardier C, Beaton D. Cross-cultural adaptation of health-related quality of life measures: literature review and proposed guidelines. J Clin Epidemiol. 1993;46(12):1417-32.

22. Hopkins WG. A new view of statistics [document on the Internet]. 2000 [cited 2007 Aug]. Available from: http://www.sportsci. org/resource/stats/index.html

23. Bell M, Bombardier C, Tugwell P. Measurement of functional status, quality of life, and utility in rheumatoid arthritis. Arthritis Rheum. 1990;33(4):591-601.

24. Basson R. Women's sexual dysfunction: revised and explanded definitions. CMAJ. 2005; 172(10):1327-33. Review.

25. 25. Hartmann U, Philippsohn S, Heiser K, Rüffer-Hesse_ C. Low sexual desire in midlife and older women: personality factors, psychosocial development, present sexuality. Menopause. 2004; 11 (6 Pt 2):726-40.

26. Basson R, Leiblum SL, Brotto, L, Derogatis L, Fourcroy J, Fugl-Meyer $\mathrm{K}$, et al. Definitions of women's sexual dysfunction reconsidered: advocating expansion and revision. J Psychosom Obstet Gynecol. $2003 ; 24(4): 221-9$. 